\author{
Military Technical College \\ Kobry Elkobbah, \\ Cairo, Egypt
}

\author{
$5^{\text {th }}$ International Conference \\ on Electrical Engineering \\ ICEENG 2006
}

\title{
STABLE NONLINEAR PREDICTIVE CONTROL FOR FAST CONSTRAINED SYSTEMS
}

\author{
Ahmed $^{*}$ M. Youssef
}

\begin{abstract}
Despite the good properties that the Nonlinear Quadratic Gaussian Predictive Control (NLQGPC) technique offers such as the low computational burden and the high performance when dealing with models with fast dynamics and input constraints, it suffers from unproven stability properties. In this paper, the recently introduced Control Lyapunov Function based satisficing approach is combined with the NLQGPC approach. This renders guaranteed asymptotic stability whilst retaining the good properties of the NLQGPC.
\end{abstract}

\section{Key Words}

State-dependent, Nonlinear Quadratic Gaussian Predictive Control, Satisficing, Control Lyapunov Function.

\section{INTRODUCTION}

There has been a lot of interest recently in the derivation of control laws that include process constraints and nonlinearities by both industry and academia. This is necessary because almost all of the physical systems in science and engineering are nonlinear in nature. Besides the nonlinearities, all real processes are subject to constraints, which may also introduce nonlinearities into the closed-loop system. Unfortunately the design of linear controllers for systems with strong nonlinearities often leads to an unsatisfactory control behavior. This motivates the need for nonlinear control design techniques, which employs the nonlinear model directly in the controller calculation, without the need for local linearization about an operating point.

Model Based Predictive Control (MBPC) is a form of control in which, at each instant of time, a performance index is minimized obtaining an optimal control sequence [1]. Only the first element of this sequence is applied to the plant. The major advantage of this type of control is its ability to account for hard constraints. Thus, by using a nonlinear predictive control design method, two objectives can be met: controlling processes in the presence of nonlinearities and dealing with hard constraints.

\footnotetext{
* Egyptian Armed Force
} 
In earlier studies on the theory of Model Based Predictive Control, the technique of quadratic programming was employed to solve the open-loop optimal control problem with constraints. This results in a rapidly growing computational burden with the number of decision variables. These earlier schemes were therefore applied only to slow processes. This suggests the need for less computationally complex nonlinear predictive control methods that can be applied to models of systems with fast dynamics.

The formulation of state-dependent state space equations, as a description of nonlinear systems, has been utilized to extend the Linear Quadratic Gaussian (LQG) approach to nonlinear systems in the framework of multi-step cost function based predictive control. This approach is called the NLQGPC [2].

Unlike other nonlinear predictive control methods, NLQGPC utilizes an explicit optimal control law that can be solved analytically, removing the need for quadratic programming. This reduces the computations and allows the controller to deal with models of systems with fast dynamics [3]. The problem of dealing with input constraints can be solved by approximating these constraints by means of smooth limiting function and including them into the dynamics of the plant. A real model of fighter aircraft has been used to examine the strengths of the NLQGPC, where the results showed that the controls based on solutions to this approach offer high performance when applied to models of systems with fast constrained dynamics [4]. Despite these attributes that make the NLQGPC an effective practical tool for the control of many systems, it suffers from the lack of guaranteed stability properties.

Despite the theory put forward by Lyapunov nearly a century ago, the fundamental problem in nonlinear systems remains the search for control laws that guarantee stability. It is well known that Lyapunov theory deals with dynamical systems without inputs. For this reason, it has traditionally been applied only to closed-loop control systems. That is, systems for which the input has been eliminated through the substitution of a predetermined feedback control. However, some recent papers proposed using Lyapunov function candidates in feedback design itself by making the Lyapunov derivative negative when choosing the control. Such ideas have been made precise with the introduction of the concept of a Control Lyapunov Function (CLF) for systems with a control input [5]. It has been shown in $[6,7]$ that the existence of a CLF for a system is equivalent to the existence of a globally asymptotically stabilizing control law $u$ everywhere, except possibly at $x=0$.

An approach recently introduced for cost-benefit analysis to decision making problems, called "Satisficing", has been combined with the properties of CLFs to construct a CLF-based control method [8], which generates a state-dependent set of controls that render the closedloop system stable with respect to a known CLF. This approach has been used in a number of papers to guarantee stability for nonlinear continuous time systems [8, 11, 12].

The main contribution of this paper is to extend the satisficing approach to the discrete-time nonlinear system case. That is, to ensure the stability of any nonlinear discrete control design technique. The NLQGPC technique is then augmented with the satisficing approach by projecting the controls based on solutions to the NLQGPC point-wise onto the stabilizing satisficing set, which renders guaranteed asymptotic stability while retaining the good properties of the NLQGPC.

The paper is organized as follows: Section 2 gives a description of the NLQGPC technique, with a demonstration of how the input constraints can be approximated by means of smooth 
limiting functions, and incorporated into the dynamics of the plant. Section 3 describes the satisficing approach and the projecting of the NLQGPC onto the satisficing set. The F-8 fighter aircraft nonlinear model, as a real model of fast dynamics, is used to demonstrate the performance of the resulting NLQGPC-satisficing controller in Section 4. Finally, this paper concludes with a brief summary in Section 5.

\section{NONLINEAR QUADRATIC GAUSSIAN PREDICTIVE CONTROL}

Consider a nonlinear continuous time system of the form:

$$
\begin{aligned}
& \dot{x}(t)=f_{1}(x(t))+f_{2}(x(t), u(t)) u(t) \\
& y(t)=f_{3}(x(t))
\end{aligned}
$$

where $f_{1}$ is a vector of size $n_{x}, f_{2}$ is a matrix of size $n_{x} \times n_{u}, f_{3}$ is a vector of size $n_{y}$.

Equation (1) can sometimes be transformed into an alternative representation, with a linearlike structure having State-Dependent Coefficients (SDC) in the following form:

$$
\begin{aligned}
& \dot{x}(t)=A(x(t)) x(t)+B(x(t), u(t)) u(t) \\
& y(t)=C(x(t)) x(t)
\end{aligned}
$$

where $A$ is a matrix of size $n_{x} \times n_{x}, B=f_{2}, C$ is a matrix of size $n_{y} \times n_{x}$.

At each iteration, the predictions of the future state and control signal may be used, instead of the future state and control which are not available, to recalculate the state-dependent statespace matrices within a prediction horizon $N$. These matrices are discretized with the sampling period $T_{s}$ and integral action is incorporated. To simplify notation, the final matrices are denoted as $A_{n}, B_{n}, C_{n}$. Thus, by computing a prediction of the future trajectory, the nonlinear system response may be approximated by a known time-varying linear system. From these future state-space matrices, the following matrices can be calculated:

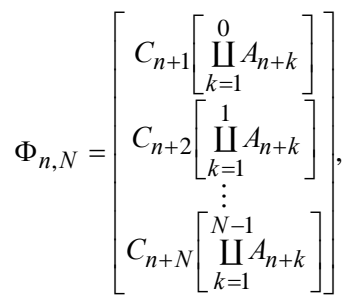

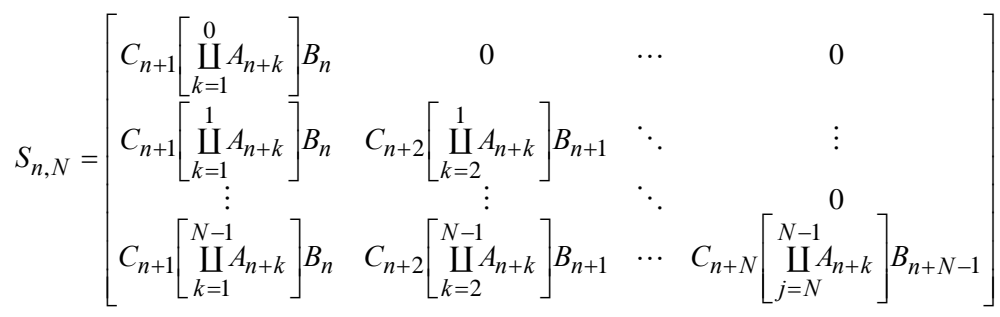


where

$$
\left[\begin{array}{l}
\bigsqcup_{k=l}^{n} A_{k}
\end{array}\right]=\left\{\begin{array}{cc}
A_{n} A_{n-1} \cdots A_{l} & \text { if } l \leq n \\
I & \text { if } l>n
\end{array}\right.
$$

\subsection{Cost Minimization Problem}

The infinite performance index to be minimized is defined as follows:

$$
\begin{aligned}
& J_{n}=\lim _{T \rightarrow \infty}\left\{\frac{1}{T+1} \sum_{k=n}^{n+T} J_{k}\right\} \\
& J_{k}=\sum_{j=1}^{N}\left\{\left(r_{k+j}-y_{k+j}\right)^{T} \Lambda_{E}^{j}\left(r_{k+j}-y_{k+j}\right)\right\}+\sum_{j=0}^{N-1}\left\{u_{k+j}{ }^{T} \Lambda_{U}^{j} u_{k+j}\right\}
\end{aligned}
$$

It is assumed that after the prediction horizon $N$, the state-space model matrices remain constant. Therefore, it is possible to obtain a solution for the Algebraic Riccati Equation (ARE) at time $n+N-1$ and to use it as a steady-state solution for solving the Difference Riccati Equation (DRE) backwards from $n=n+N-1$ to $n=1$. By using the solution of the DRE at $n=1$, the optimal control may be calculated as follows:

$$
\begin{aligned}
U_{n, N}= & -\left(\Lambda_{U}+S_{n, N}{ }^{T} \Lambda_{E} S_{n, N}+\beta_{n}^{T} \tilde{H}_{n+1}^{1} \beta_{n}\right)^{-1} . \\
& {\left[\left(S_{n, N}{ }^{T} \Lambda_{E} \Phi_{n, N} A_{n}+\beta_{n}^{T} \tilde{H}_{n+1}^{1} A_{n}\right) x_{n}+\left(\beta_{n}^{T} \tilde{H}_{n+1}^{2} \Theta_{n, N}-S_{n, N}{ }^{T} \Lambda_{E}\right) R_{n+1, N}\right] }
\end{aligned}
$$

where $\Lambda_{E}^{i} \geq 0, \quad i=1 \ldots N$ and $\Lambda_{U}^{j}>0, \quad j=1 \ldots N-1$ are weighting matrices and $N$ is a positive integer greater than or equal to unity. The vector $R_{n+1, N}$ is a vector containing current and future values of set point $r_{n}$. $\beta_{n}$ is constructed from the matrix $B_{n}$ as follows:

$$
\beta_{n}{ }^{T}=\left[\begin{array}{llll}
B_{n}{ }^{T} & 0 & \cdots & 0
\end{array}\right]
$$

$\Theta$ is the transition matrix for the reference signal. i.e. it is assumed that:

$$
R_{n+1, N}=\Theta_{n, N} \cdot R_{n, N}
$$

where $\tilde{H}_{n+1}^{1}$ and $\tilde{H}_{n+1}^{2}$ are the solutions of the coupled Riccati equations. See [9] for a detailed derivation of this algorithm.

As with most MBPC techniques, at each iteration, only the first element of the calculated control vector is applied to the plant, but unlike other MBPCs, the remaining part of the control vector, referred to as the "tail" [10], is also used to predict future states and consequently system matrices by employing an appropriate numerical integration method.

\subsection{Input Constraints}


The standard way of handling the constraints in an optimization problem is to add them to the cost function using Lagrange multipliers, which results in an unconstrained optimization problem. It is well known that the solution of the ARE is equivalent to the solution of the Lagrange multiplier approach for solving the LQ problem, with the constraint equations being determined by the dynamics of the plant. The approach here is similar since input constraints are incorporated into the dynamics of the plant in a state-dependent structure form. This enables the constraints to be taken into account, whilst preserving the problem as being unconstrained from the ARE point of view.

Different types of actuator constraints have been investigated in [4], where it was shown that the magnitude saturation constraint represents the most significant nonlinearity in a control system, since it is a common term in the other constraints equations. Limiting functions that possess a smooth transition to the saturation from 0 to 1 (for instance, the logarithmic sigmoid) have been used to approximate the saturation of actuators that have a limited range of operation from zero to a certain value $\alpha$. Other smoothing limiting functions that map the interval $(-\infty, \infty)$ onto $(-1,1)$ have been used to approximate the magnitude saturations limited from $-\alpha$ to $\alpha$, such as the hyperbolic tangent tanh function, and the error function erf. By obtaining an approximation to the magnitude saturation function, input constraints such as actuator saturation, rate-limited actuator, and actuator dead-zone, can be expressed as:

$$
u_{\text {out }}=l_{s}\left(u_{\text {in }}\right)
$$

where $u_{\text {in }}$ and $u_{\text {out }}$ are the input and the output of the actuator, respectively. $l_{s}(u)$ denotes the smooth limiting function. Then the system (2) can be rewritten as follows:

$$
\begin{aligned}
\dot{x} & =A(x(t)) x(t)+B(x(t)) u_{\text {out }} \\
& =A(x(t)) x(t)+B(x(t)) \frac{l_{s}\left(u_{i \text { in }}\right)}{u_{\text {in }}} u_{\text {in }} \\
& =A(x(t)) x(t)+\aleph\left(x(t), u_{\text {in }}(t)\right) u_{\text {in }}
\end{aligned}
$$

where $\aleph\left(x(t), u_{i n}(t)\right)=B(x(t)) \frac{l_{s}\left(u_{i n}\right)}{u_{i n}}$ is the new input matrix of the augmented system. For non-strictly proper systems, the direct transmission matrices $D(s)$ will be treated as the input matrices $B(s)$. By this approach, the input constraints are included into the dynamics of the plant in a state-dependent state-space form.

\section{SATISFICING}

In this section, a straightforward technique for designing a stabilizing controller, once a Control Lyapunov Function (CLF) has been found, is presented. Consider an affine discrete nonlinear system with a quadratic discrete CLF:

$$
x_{k+1}=f\left(x_{k}\right)+B\left(x_{k}\right) u
$$




$$
V\left(x_{k}\right)=x_{k}^{T} P x_{k}
$$

where $u \in \mathfrak{R}^{\mathrm{m}}, f(0)=0, P$ is positive definite matrix.

Definition 1: A $C^{l}$ function $V\left(x_{k}\right): \mathfrak{R}^{\mathrm{n}} \rightarrow \mathfrak{R}$ is said to be a discrete-time CLF for the system (12), if $V\left(x_{k}\right)$ is positive definite, unbounded, and if the increment $\left.\Delta V\left(x_{k}, u\right)\right|_{u=u_{k}}<0$ for all $x_{k} \neq 0$.

An approach recently introduced for decision-making problems, called Satisficing, has been used to develop a CLF-based control method for ensuring the closed-loop stability of nonlinear systems. The word "Satisficing" means, "deciding what constitutes a satisfactory outcome rather than an optimal one and then looking for ways to achieve it”. This method has been used in a number of papers to guarantee stability for nonlinear continuous time systems $[8,11,12]$. The objective of this section is to extend the satisficing approach to the discretetime nonlinear system case. That is, to ensure the stability of any nonlinear discrete control design technique.

The CLF-based satisficing approach is based on a point-wise cost-benefit trade-off, where benefits are defined in terms of the CLF, and costs reflect a penalty on the control effort and the state. The satisficing set $S$ as defined in [11] is the set of control values such that the benefits are greater than the costs. Therefore in the discrete-time case, $S$ can be expressed as follows:

$$
S(x, b)=\left\{u:-\Delta V(x, u) \geq \frac{1}{b}\left(l(x)+u^{T} R u\right)\right\}
$$

where $R=R^{T}>0, b$ is a selectivity index, and $l(x)$ is a non-negative, locally Lipschitz function that represent the design parameters. Therefore, the satisficing set $S$ is a statedependent set of controls that renders the closed-loop system stable with respect to a known CLF. It is clear that the condition for membership in $S, \Delta V\left(x_{k}, u_{k}\right)<0$, is satisfied as the parameter $b$ approaches infinity.

For short, we will use $f$ and $B$ instead of $f\left(x_{k}\right)$ and $B\left(x_{k}\right)$. For such a class of systems, the increment $\Delta V$ can be written as:

$$
\begin{aligned}
\Delta V\left(x_{k}, u_{k}\right) & =V\left(x_{k+1}\right)-V\left(x_{k}\right) \\
& =\left\{V(f)+\left.\int_{0}^{u_{k}} \frac{\partial V(\xi)}{\partial \xi}\right|_{\xi=F\left(x_{k}, v\right)} \frac{\partial F\left(x_{k}, v\right)}{\partial v} d v\right\}-V\left(x_{k}\right) \\
& =f^{T} P f-x_{k}^{T} P x_{k}+2 \int_{0}^{u_{k}}\left[v B^{T} P B+B^{T} P f\right] d v<0 \\
& =f^{T} P f-x_{k}^{T} P x_{k}+2 f^{T} P B u_{k}+u_{k}^{T} B^{T} P B u_{k}
\end{aligned}
$$

Theorem 1 [14]: $V\left(x_{k}\right)=x_{k}{ }^{T} P x_{k}$ is a discrete-time CLF for the system (12) if and only if there exists $P>0$ satisfying: 


$$
f^{T} P f-x^{T} P x-f^{T} P B\left[B^{T} P B\right]^{-1} B^{T} P f<0
$$

then the control law $u\left(x_{k}\right) \in\left(0, u^{*}\left(x_{k}\right)\right]$ globally asymptotically stabilizes the origin of the discrete time dynamics (12).

where

$$
u^{*}\left(x_{k}\right)=-\left(B^{T} P B\right)^{-1} B^{T} P f
$$

Proof : see [14].

From Equations (16) and (17), we obtain:

$$
f^{T} P f-x^{T} P x-\left(-f^{T} P B\right) u<0
$$

Hence, the satisficing set of stabilizing control values is only dependent on the vector $-B^{T} P f$. More precisely as can be shown in Figure 1, the satisficing set is a region of the control space at every fixed $\mathrm{x}$ that contains all control values which render $\Delta V$ negative, and it is an open half space, bounded by the hyperplane which lies perpendicular to the vector $-B^{T} P f$.

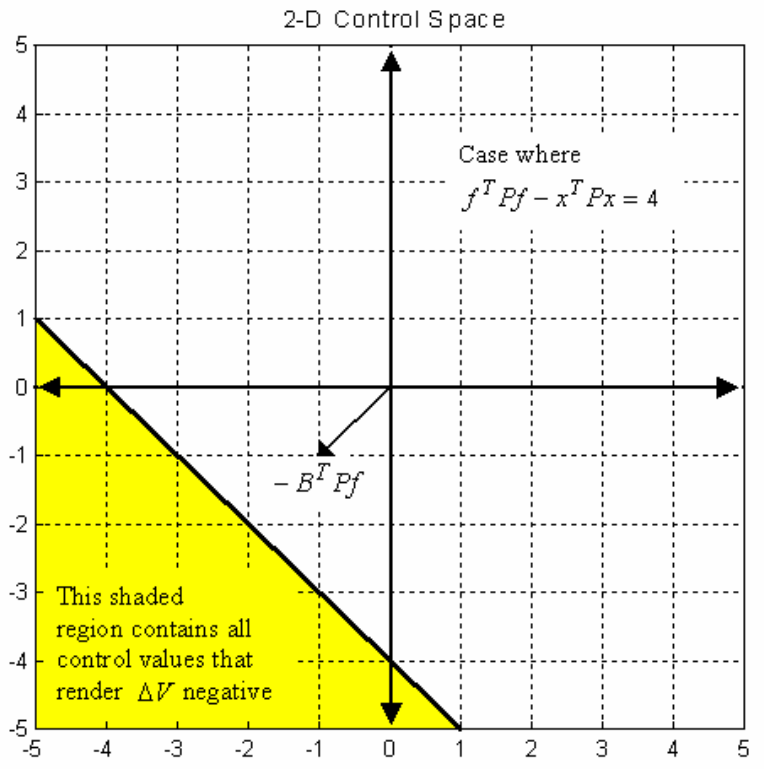

Figure 1: Satisficing set in a 2-D control space

Note from Equation (15) that $\Delta V$ is a convex function since $\frac{\partial^{2}(\Delta V)}{\partial u^{2}} \geq 0, u \in \mathfrak{R}^{\mathrm{m}}$, and hence, $\Delta V$ has a global minimum. Now, setting $\frac{\partial(\Delta V)}{\partial u}=0$ it follows that:

$$
\bar{u}=-\frac{1}{2}\left(B^{T} P B\right)^{-1} B^{T} P f
$$

Therefore, an arbitrary discrete control value can be written as: 


$$
u=-\beta(x)\left(B^{T} P B\right)^{-1} B^{T} P f+\xi(x)
$$

where $\xi(x)$ is orthogonal to the vector $-B^{T} P f$.

Theorem 2: An arbitrary discrete-time control law $u=-\beta(x)\left(B^{T} P B\right)^{-1} B^{T} P f+\xi(x)$ with $u(0)=0$ is asymptotically stabilizing (lie in $S$ ) with respect to the known discrete time CLF, $V(x)$, if and only if:

$$
\beta>\frac{f^{T} P f-x^{T} P x}{f^{T} P B\left(B^{T} P B\right)^{-1} B^{T} P f}
$$

Proof:

$$
\begin{array}{ll} 
& \Delta V<0 \\
\Leftrightarrow & f^{T} P f-x^{T} P x+f^{T} P B u<0 \\
\Leftrightarrow & f^{T} P B u<-\left(f^{T} P f-x^{T} P x\right) \\
\Leftrightarrow & -\beta f^{T} P B\left(B^{T} P B\right)^{-1} B^{T} P f<-\left(f^{T} P f-x^{T} P x\right) \\
\Leftrightarrow & \beta>\frac{f^{T} P f-x^{T} P x}{f^{T} P B\left(B^{T} P B\right)^{-1} B^{T} P f}
\end{array}
$$

Finally, the procedure for point-wise projection of the NLQGPC (as an example of discretetime control design technique) control value onto $S$ is as follows:

If $u_{N L Q G P C}$ is an arbitrary NLQGPC control value and $\beta$ is defined as:

$$
\beta=\frac{-u_{N L Q G P C}^{T} B^{T} P f}{f^{T} P B\left(B^{T} P B\right)^{-1} B^{T} P f}
$$

then $\beta>\frac{f^{T} P f-x^{T} P x}{f^{T} P B\left(B^{T} P B\right)^{-1} B^{T} P f}$ implies $u_{N L Q G P C} \in S$.

Otherwise, the augmented control:

$$
u_{\text {aug }}=u_{N L Q G P C}-\left(\frac{f^{T} P f-x^{T} P x}{f^{T} P B\left(B^{T} P B\right)^{-1} B^{T} P f}-\beta+\tau\right)\left(B^{T} P B\right)^{-1} B^{T} P f
$$

where $\tau$ is some very small number, represents the projection of $u_{N L Q G P C}$ onto $S$. The flow chart of the satisficing algorithm is shown in Figure 2. 


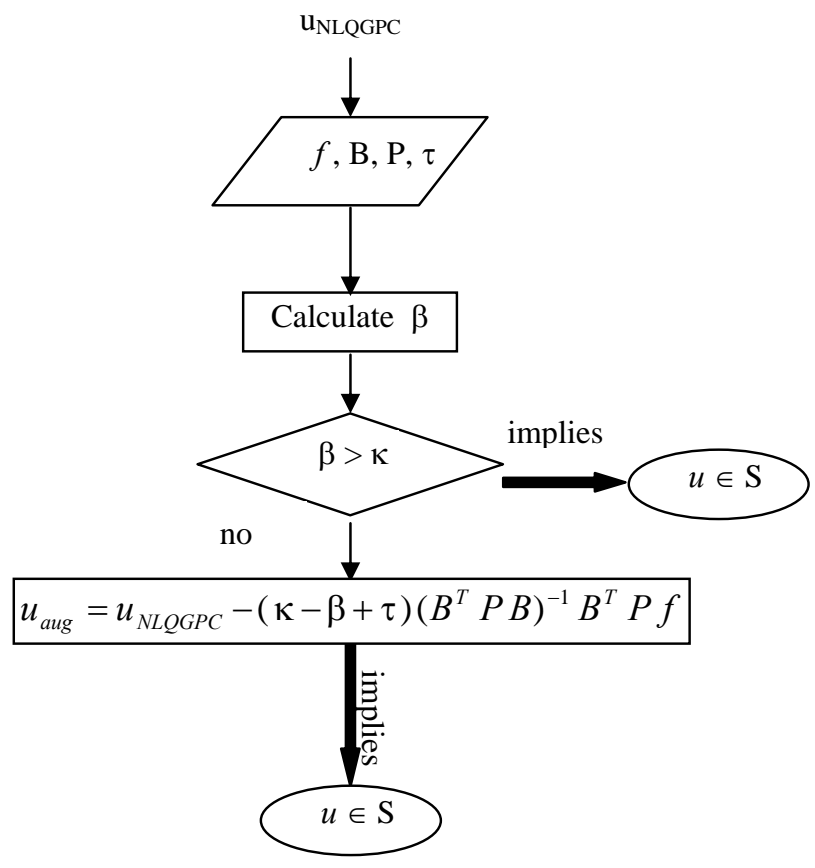

Figure 2: Satisficing algorithm flow chart

where $\kappa=\frac{f^{T} P f-x^{T} P x}{f^{T} P B\left(B^{T} P B\right)^{-1} B^{T} P f}$

\section{CONTROL OF F-8 AIRCRAFT}

This section deals with controlling the F-8 aircraft as a real model of fast dynamics. The nonlinear dynamical model of the F-8 fighter aircraft, adopted from [13], may be given in state-space representation as:

$$
\begin{aligned}
\dot{x}_{1}= & -0.877 x_{1}+x_{3}-0.088 x_{1} x_{3}+0.47 x_{1}^{2}-0.019 x_{2}^{2}-x_{1}^{2} x_{3}+3.846 x_{1}^{3}-0.215 u \\
& +0.28 x_{1}^{2} u+0.47 x_{1} u^{2}+0.63 u^{3}, \\
\dot{x}_{2}= & x_{3}, \\
\dot{x}_{3}= & -4.208 x_{1}-0.396 x_{3}-0.47 x_{1}^{2}-3.564 x_{1}^{3}-20.967 u+6.265 x_{1}^{2} u+46 x_{1} u^{2}+61.4 u^{3}
\end{aligned}
$$

where $x_{1}$ is the angle of attack in radians, $x_{2}$ is the pitch angle in radians, $x_{3}$ is the pitch rate in radians/s, and the control input $u$ is the elevator deflection angle in radians. The set of equations (24) represents the short-period approximation mode, which has a relatively short time constant. For flight safety, stabilization of this mode is required using a feedback control, since the pilot cannot control this mode. Therefore, the task is to stabilize the aircraft to the origin while fulfilling the following input constraint:

$$
|u| \leq 0.05236 \mathrm{rad}
$$




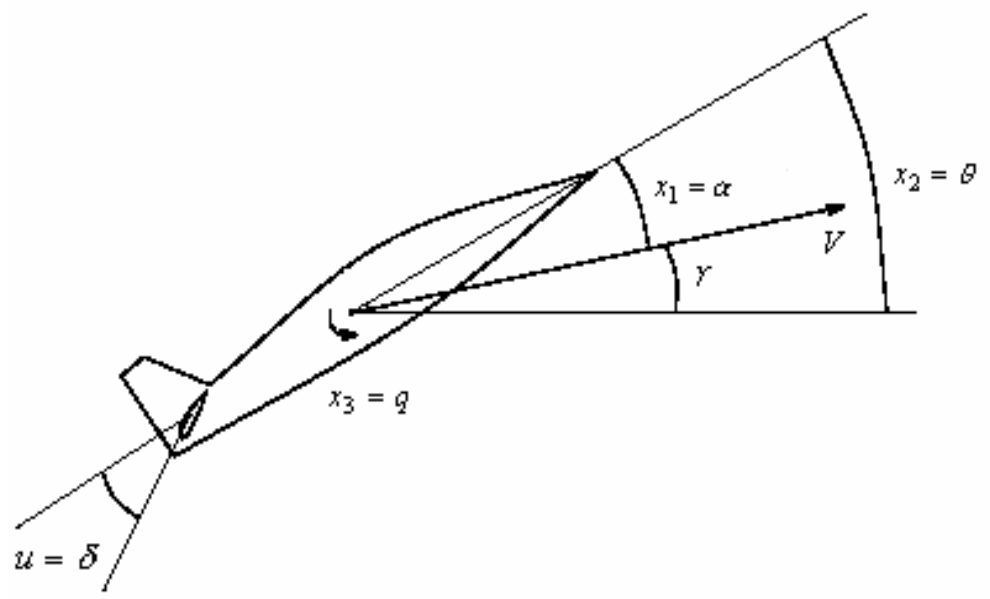

Figure 3: Illustration of longitudinal aircraft variables.

First, (24) is transformed into a state-dependent state-space representation form (2), as follows:

$$
A(x(t))=\left[\begin{array}{ccc}
a_{11} & a_{12} & 1 \\
0 & 0 & 1 \\
a_{31} & 0 & a_{33}
\end{array}\right], \quad B(x(t))=\left[\begin{array}{c}
b_{1} \\
0 \\
b_{3}
\end{array}\right]
$$

where $a_{11}=-0.877-0.088 x_{3}+0.47 x_{1}-x_{1} x_{3}+3.846 x_{1}^{2}$,

$$
\begin{aligned}
& a_{12}=-0.019 x_{2}, \\
& a_{31}=-4.208-0.47 x_{1}-3.564 x_{1}^{2}, \\
& a_{33}=-0.396, \\
& b_{1}=-0.215+0.28 x_{1}{ }^{2}+0.47 x_{1} u+0.63 u^{2}, \\
& b_{3}=-20.967+6.265 x_{1}{ }^{2}+46 x_{1} u+61.4 u^{2} .
\end{aligned}
$$

The short-period mode is displayed in the motion of the aircraft angle of attack $x_{1}$ and the pitch rate $x_{3}$, therefore the output matrix $C$ is given as:

$$
C=\left[\begin{array}{lll}
1 & 0 & 0 \\
0 & 0 & 1
\end{array}\right]
$$

The error function erf is used as a smooth limiting function. The input constraint is included into the plant dynamics as in equation (11), where the input matrix of the augmented system is given as:

$$
\aleph(x(t))=B(x(t)) \frac{0.05236 \operatorname{erf}\left(\frac{u}{0.05236}\right)}{u}
$$

It is well known that for linear systems, if the pair $(A, B)$ is controllable, control design and search for Lyapunov function are done through the backward procedure as follows: given positive definite matrices $Q$ and $R$, there is a unique positive definite matrix $P$ satisfying the ARE, then the Lyapunov function is $V(x)=x^{T} P x$. Since by using state dependent state- 
space form the nonlinear system is approximated by a time-varying linear system, therefore, the candidate CLF is given as:

$$
V(x)=x^{T} \Psi x
$$

where the positive definite matrix $\Psi$ is the average of the solutions of the ARE(s) over the whole simulation time (this doesn't always work for all systems of course).

The NLQGPC is designed based on equation (7), with $N=6, \Lambda_{E}=\left[\begin{array}{cc}0.01 & 0 \\ 0 & 0.015\end{array}\right], \Lambda_{U}=1$, and the sampling time $T_{s}=250 \mathrm{~ms} . \tau$ is chosen to be 0.001 . The closed loop responses of the system from the initial condition $x^{T}(0)=\left[\begin{array}{llll}0.4655 & 0 & 0\end{array}\right]^{T}$ are shown in Figures 4-5, while Figure 6 shows the trajectory of the elevator deflection.

The response due to the constrained NLQGPC (dashed line) is almost identical to the response due to the NLQGPC-satisficing controller (solid line) except for few points, which lie outside the satisficing set $S$. These points are pulled inside $S$, by the satisficing method, which explains the very small deviation between responses. Although the two responses coincide with each other, the closed-loop response due to the NLQGPC-satisficing controller is guaranteed to be asymptotic stable, whereas the closed-loop response due to the constrained NLQGPC has no such guarantees.

Figure 7 shows the norm of the difference between the constrained NLQGPC and the constrained NLQGPC-satisficing controller. The difference is very small which proves that the good properties of the constrained NLQGPC are retained while the analytical properties of the satisficing approach have been added. Asymptotical stability is proven by using different initial conditions as shown in Figure 8, where as the trajectories converge to the origin as $t \rightarrow$ $\infty$.

\section{CONCLUSIONS}

In this paper, a discrete-time CLF-based control method is presented for determining closedloop stability under the recently developed satisficing approach. A solution for the stability problem of the NLQGPC strategy utilizing this method is proposed. A simulation of a nontrivial example, of an F-8 fighter aircraft, provides a demonstration of the results, which shows that the good properties of the NLQGPC technique are retained whilst offering guarantees to asymptotically stabilize the closed-loop system. The resulting augmented controller has many attributes that make it a more effective practical tool for controlling systems of fast-constrained dynamics.

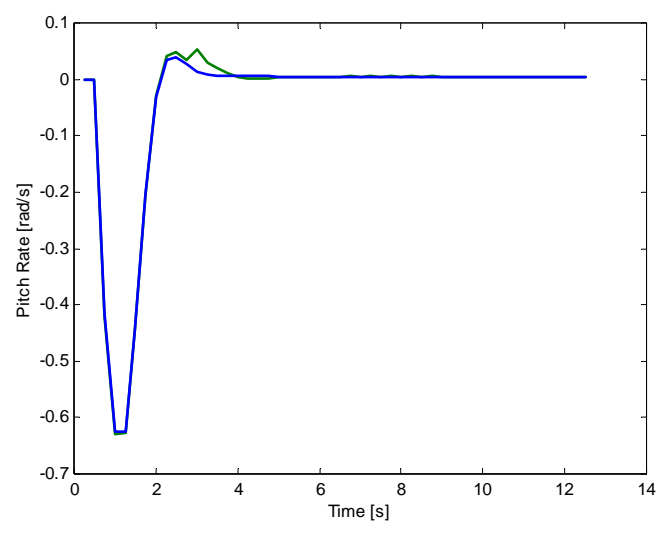




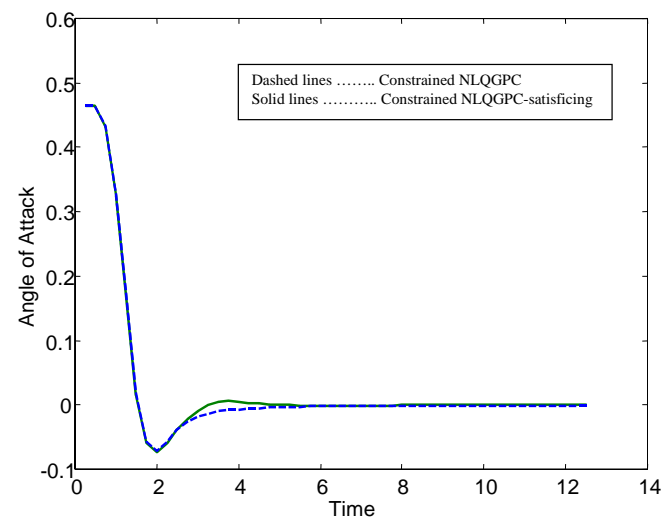

Figure 4: Angle of attack response

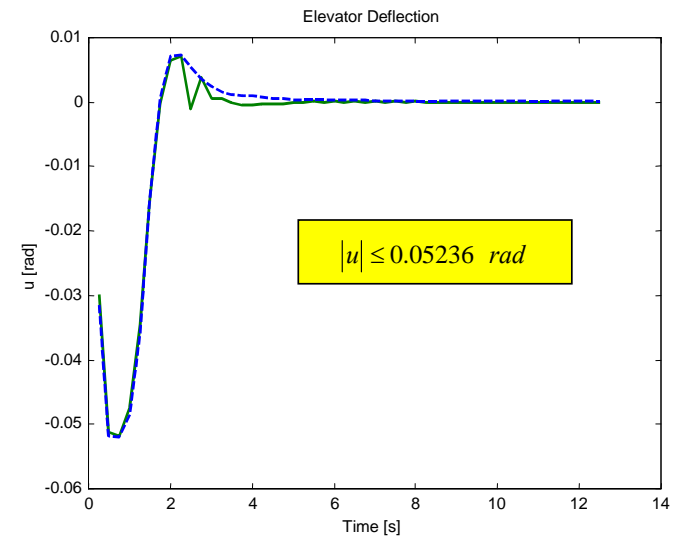

Figure 6: The elevator deflection
Figure 5: Pitch-rate response

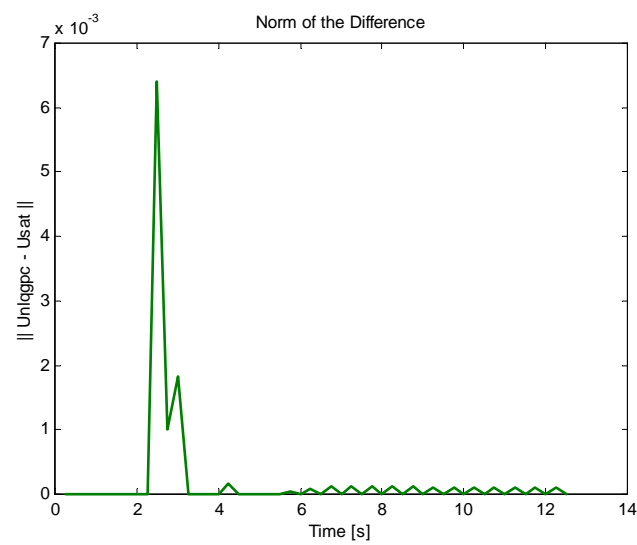

Figure 7: The norm of the difference

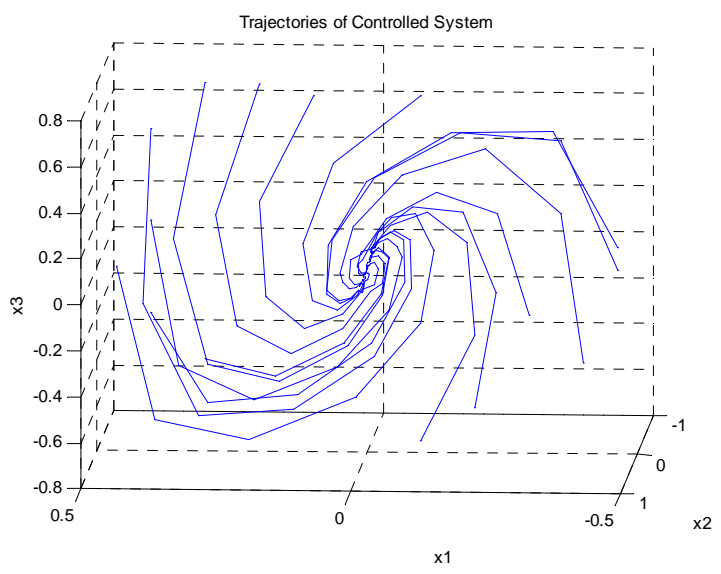

Figure 8: The trajectories of the controlled system to various initial conditions

\section{REFERENCES}


[1] Grimble M. J., Industrial Control Systems Design, John Wiley, Chichester, 2000.

[2] Grimble M. J., Ordys A. W., Non-linear Predictive Control for Manufacturing and Robotic Applications, IEEE Conference on Methods and Models in Automation and Robotics, Poland, August 2001.

[3] Youssef A. M., Grimble M. J., Ordys A. W., Dutka A., and Anderson D., Robust Nonlinear Predictive Flight Control, European Control Conference, Cambridge, UK, 2003.

[4] Youssef, A. M., Ordys, A. W., and Grimble, M. J., Nonlinear Predictive Control for Fast Constrained Systems, Proceedings of the 10th IEEE International Conference on Methods and Models in Automation and Robotics (MMAR), pp. 463-468 2004.

[5] Ghandhari M., Andersson, G., and Hiskens I. A., Control Lyapunov Functions for Controllable Series Devices, IEEE Trans. on Power Systems, vol. 16, no. 4, November 2001.

[6] Artstein Z., Stabilization with Relaxed Control, Nonlinear Analysis, Theory, Methods, and Applications, vol. 7, no. 11, pp. 1163-1173, 1983.

[7] Sontag E. D., A Universal Construction of Artstein's Theorem on Nonlinear Stabilization, System \& Control Letters, vol. 13, pp. 117-123, 1989.

[8] Goodrich M. A., Stirling W. C., and Frost R.L., A Theory of Satisficing Decisions and Control, IEEE Trans. Syst., Man, Cybern. A, vol. 28, pp. 763-779, November 1998.

[9] Grimble M. J., Ordys A. W., and Dutka A., Nonlinear Quadratic Gaussian Predictive Control, Submitted for publication in Transactions of the ASME.

[10] Kouvaritakis B., Cannon M., and Rossiter J. A., Non-linear model based predictive control, Int. J. Control, vol. 72, no. 10, pp. 919-928, 1999.

[11] Curtis J. W. and Beard R. W., Ensuring Stability of State-dependent Riccati Equation Controllers Via Satisficing, IEEE Conference on Decision and Control, Nevada, USA, pp. 2645-50, December 2002.

[12] Beard R. W., Young B., and Stirling W. C., Nonlinear Regulation Using the Satisficing Paradigm, In Proceedings American Control Conference, pages 4258-4263, 2001.

[13] Kaya C. Y. and Noakes J. L., Computations and Time-Optimal Controls, Optimal Control Applications \& Methods 17, pp.171-185, 1996.

[14] Amicucci, G.L., Monaco, S., and Normand-Cyrot D., Control Lyapunov Stabilization of Affine Discrete-time Systems, In Proc. IEEE Conf. Dec. Contr., pp. 923-924, San Diego, CA, 1997. 